\title{
Moderate-Intensity Walking Training Improves Depressive Symptoms and Pain in Older Adults with Good Quality of Life: A Controlled Randomized Trial
}

Silvio Lopes Alabarse, ${ }^{1 \oplus}$ Hélio José Coelho Júnior, ${ }^{2 \oplus}$ Ricardo Yukio Asano, ${ }^{3 \oplus}$ Braulio Luna Filho, ${ }^{1 \oplus}$ Wagner Correa Santos, ${ }^{\circledR}$ Japy Angelini Oliveira Filho ${ }^{\circledR}$

Universidade Federal de São Paulo (Unifesp), ${ }^{1}$ São Paulo, SP - Brazil

Universidade de Campinas (Unicamp), ${ }^{2}$ Campinas, SP - Brazil

Universidade de São Paulo (USP), ${ }^{3}$ São Paulo, SP - Brazil

\section{Abstract}

Background: Walking training can be an adequate choice to improve physical and psychological conditions in the elderly. Studies have reported positive changes in the quality of life, depressive symptoms and pain. However, baseline characteristics of volunteers have been controlled, and some of previous studies have not investigated these parameters concomitantly.

Objectives: To assess the effects of moderate-intensity walking on quality of life, depressive symptoms and physical pain in physically active elderly individuals.

Methods: Sixty-nine subjects were recruited and allocated into two groups: training group ( $\mathrm{n}=40)$ and control group ( $\mathrm{n}=29$ ). All were evaluated for quality of life, depressive symptoms and pain. Training group underwent 40 minutes of walking (50-70\% of maximum heart rate), 3 days a week for 12 weeks. For statistical analysis, we used the Kolmogorov-Smirnov test, Student's t-test and Split-Plot ANOVA with Bonferroni post hoc, Pearson correlation. Significance level was set at $5 \%$.

Results: After 12 weeks of training, depressive symptoms and physical pain significantly reduced in the training group ( $2.7 \pm 2.4$ to $1.9 \pm 1.8$ and $4.3 \pm 3.1$ to $2.8 \pm 2.9$, respectively) compared with baseline values, and remained unchanged in the control group. There was a positive, moderate correlation between depressive symptoms and pain $(\mathrm{r}=0.30)$.

Conclusion: physically active elderly individuals with good quality of life show improved depressive symptoms after a short-term moderate-intensity walking training program. (Int J Cardiovasc Sci. 2019;32(6):553-562)

Keywords: Walking; Walking Speed; Quality of Life; Aged; Pain; Depression.

\section{Introduction}

The United Nations estimate that there will be 1.5 billion senior citizens in the world by $2050 .{ }^{1}$ Aging process is a continuous and multidimensional phenomenon that generally leads to a decrease in independence and performance of daily life activities..$^{2-4}$ Quality of life (QOL) is one of the variables affected by aging, that may be differently described even by individuals living in similar conditions, including in terms of psychological aspects, as depressive symptoms. . $^{3,5,6}$
Despite the possible influence of several factors on the level of QOL, a worse QOL is observed in patients with high prevalence of depressive symptoms and increased physical pain. This phenomenon deserves attention, as these symptoms are associated with frailty, leading to a poor prognosis. ${ }^{7}$

Physical exercise is a powerful tool to prevent or at least delay the development of deleterious effects of aging. ${ }^{8-10}$ Physical training can impact on both morphological and physical domains, causing an increase in muscle mass and strength, and improvement of cognitive and 
executive functions. ${ }^{8,9}$ Besides, physical exercise can also act as a non-pharmacological treatment for chronic degenerative conditions, such as hypertension, diabetes mellitus and osteroporosis. ${ }^{11}$

Moderate-intensity aerobic exercise has been widely recommended for the elderly, due to considerable evidence suggesting it as a safe approach, able to control cardiovascular risks. ${ }^{12,13}$ This is reinforced by the American College of Sports and Medicine (ACSM), which states that walking training can have these effects in elderly individuals, and should easily be included as part of their usual daily activities. ${ }^{14,15}$

Walking training has beneficial psychological and physical effects. ${ }^{16-18}$ Studies have reported improvement in the QOL, depressive symptoms and physical pain in elderly subjects undergoing moderate-intensity aerobic exercise protocols. ${ }^{19-22}$

However, few studies have controlled baseline characteristics of volunteers, especially for QOL, pain and depressive symptoms.

The present study aimed to investigate the effects of moderate intensity walking training on depressive symptoms and physical pain in elderly individuals with a good quality of life.

\section{Methods}

\section{Study design and ethical aspects}

This is a prospective longitudinal study aiming at investigating the effects of a 12-week exercise program including moderate-intensity walking on depressive symptoms and physical pain in elderly individuals with a good quality of life.

All volunteers were instructed about the study and then signed the consent form. The study was approved and conducted according to the Ethics Committee of the University of Sao Paulo ethical standards (approval number $0562 / 11$ ) and performed according to the Brazilian National Health Council resolution (196/96).

\section{Patients}

All patients were recruited from an elderly Community Center in São Paulo, Brazil. Inclusion criteria were: a) medical clearance from the assistant physician for participation in the study; b) age $>60$ years; c) $>150$ minutes per week of physical activity according to the International Physical Activity Questionnaire (IPAQ) recommendations; ${ }^{23} \mathrm{~d}$ ) a score between 1 and 7 in the Lequesne algofunctional index for hip and knee osteoarthritis, ${ }^{24}$ and scores $\geq 40$ in the World Health Organization Quality of Life (WHOQOL)-BREF and WHOQOL-OLD questionnaires. Exclusion criteria were absence of one or more evaluations, hormone replacement and/ or psychotropic drugs, cardiovascular disease (acute myocardial infarction, stroke, peripheral artery disease and transient ischemic attack), metabolic diseases (type 1 or type 2 diabetes mellitus), pulmonary diseases (emphysema), psychiatric or neurologic diseases (Alzheimer's or Parkinson's disease), muscular disorders, skeletal disorders, comorbidities associated with higher risk of falls, clinical diagnosis of diseases associated with physical pain (low back pain) and response rates $\geq 5$ in the short form of the Geriatric Depression Scale (GDS).

A total of 165 patients were invited to participate in the study; 96 were excluded: 22 declined to participate, 19 did not answer the invitations, and four had functional impairment. Therefore, 69 volunteers were considered eligible to participate in the study and were randomized by an assistant researcher, using a simple randomization system, a table of random numbers, and categorized in two groups - training group (TG; $\mathrm{n}=40)$ and control group $(C G ; n=29)$ (Figure 1). All volunteers were blinded to the intervention.

\section{Assessments}

All assessments were performed by an investigator who was blinded to the group. Participants were asked to refrain from exercise during the 96 hours before the measurement, and from consuming caffeinated, alcoholic and energy beverages. Although energy intake was not controlled, all volunteers were asked to maintain their usual diet during the study period. No volunteer participated in any other exercise program during the study.

All tests and experimental sessions were carried out at Unifesp Psychobiology Laboratory, under a controlled temperature between 22 and $24^{\circ} \mathrm{C}$ and relative humidity of $51 \%$. All interviews were in-person and individualized. If the interviewee did not understand the question, the interviewer repeated it slowly, until three times.

\section{WHOQoL-OLD and WHOQUoL-BREF}

The WHOQOL is an instrument recommended to assess the QOL of elderly individuals. This questionnaire is composed of 24 items, organized in six major domains. 


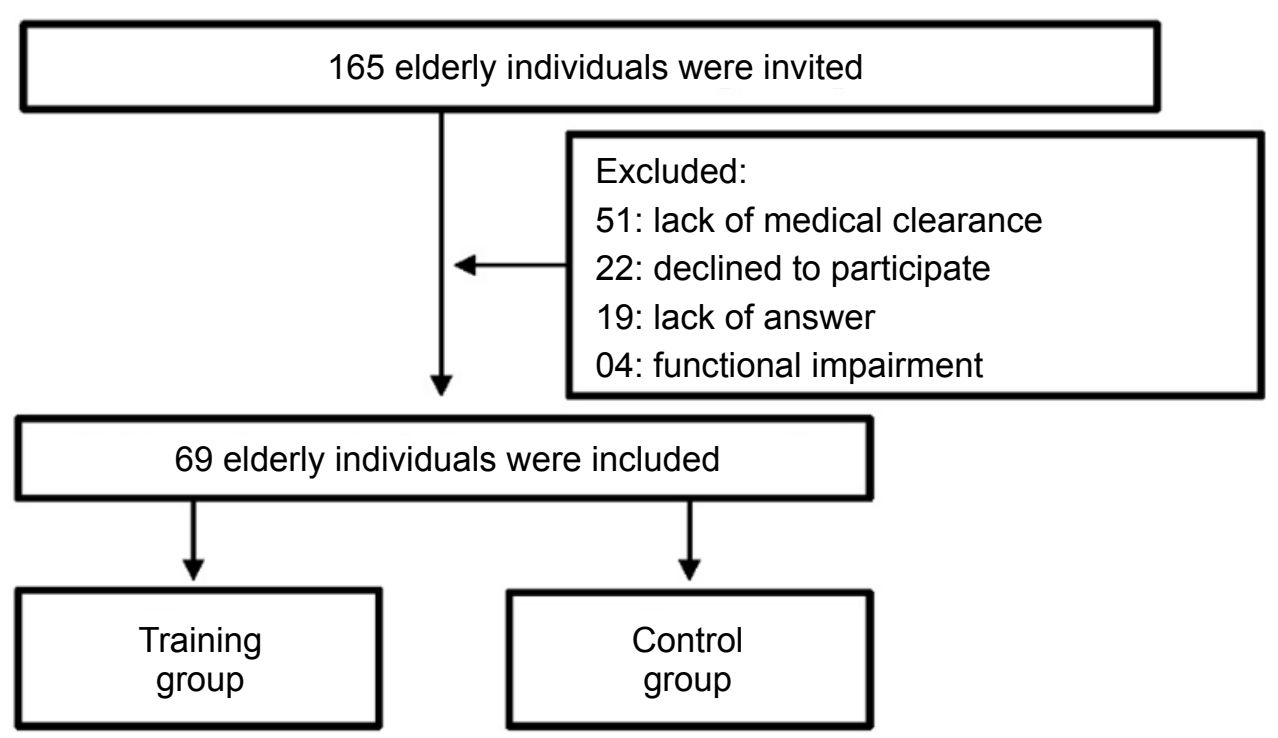

Figure 1 - Study flow chart.

The values of the six domains or the sum of the 24 questions may be combined to produce an overall score. However, there is no cut-off point, the greater the score, the better the QOL. ${ }^{25}$

Considering that the $\mathrm{WHO}$ recommends that the WHOQOL-OLD should not be administered alone, ${ }^{25}$ we used the WHOQOL-BREF also. The WHOQOLBREF is a short version of the WHOQoL-100, used in situations when the time available for application is short and when the number of respondents is large. The WHOQOL-BREF is composed of 26 questions, two of them related to QOL. The questions are organized in four domains: physical, psychological, social relationships and environment, and are positively correlated with the WHOQOL-100 overall score. ${ }^{26}$

\section{Geriatric depression scale (GDS)}

Depressive symptoms were assessed by the short version of the GDS, composed of 15 questions about feelings, and frequency of feelings in relation to life situations. The answers to the GDS questions are based on a binary code: yes or no. ${ }^{27,28}$

\section{Visual analogue scale (VAS)}

VAS is a widely used instrument to evaluate pain intensity in different populations, including the elderly. It contains a continuous scale consisting of a $10 \mathrm{~cm}$ horizontal line. The respondents place a line perpendicular to the VAS line at the point that represents their pain intensity. Quantification of pain intensity, volunteers were asked about the intensity of physical pain during the last week. A zero (0) means 'no pain' and a ten (10) means the worst pain imaginable. VAS score is positively associated with pain intensity. ${ }^{29}$

\section{Incremental exercise test}

Before the cardiac stress test, remained seated for 20 minutes. A resting electrocardiogram was performed to evaluate heart pressure, heart rate and lactate.

Then, incremental test was performed using an electronic treadmill (Life Fitness ${ }^{\circledR}$, model $9700 \mathrm{HR}^{\circledR}$, Fort Mill, Tennessee, USA), according to the modified Bruce protocol, composed of six stages of three minutes each, characterized by both speed $(2.7-6.8 \mathrm{~km} / \mathrm{h})$ and slope $\left(0-16^{\circ}\right)$ increments.

The gases produced were analyzed during the incremental test using an analyzer $\left(\right.$ Cosmed $^{\circledR}$, Cosmed Quarker $\mathrm{CPET}^{\circledR}$, Rome, Italy), previously calibrated, with a 3L syringe (flow calibration) and a standard gas mixture containing $4.9 \% \mathrm{CO}_{2}$ and $17.0 \% \mathrm{O}_{2}$ (gas calibration). Ventilation (VE), oxygen consumption $\left(\mathrm{VO}_{2}\right)$ and carbon dioxide production $\left(\mathrm{VCO}_{2}\right)$ were evaluated in the last 20 minutes of each 3-minute stage. 
Heart rate (HR) was continuously measured (Oregon Scientific ${ }^{\circledR}, \mathrm{SE128}{ }^{\circledR}$, Portland, Oregon, USA). Peak $\mathrm{VO}_{2}$ and maximum HR (MaxHR) were recorded at physical exertion of patients.

All volunteers met two criteria for peak $\mathrm{VO}_{2}$ : a) respiratory exchange ratio (RER) $\geq 1.1 ; b)$ MaxHR at least equal to $90 \%$ of the maximum predicted for age, using the Jones equation ( $0.65 \times$ age -210$)$. The electrocardiographic patterns were recorded and analyzed by a cardiologist during all the test.

\section{Training group (TG)}

The intensity, frequency and duration of the aerobic training sessions were conducted according to the ACSM and the American Heart Association (AHA) recommendations. Each participant underwent 36 walking sessions, which occurred three days a week for 12 weeks, with a minimum of 48 hours of recovery between the sessions. Each session consisted of five minutes of warm-up, which involved movements of the whole body; 30 minutes of continuous walking at moderate intensity $-50-70 \%$ of MaxHR, established at the stress test previously performed on the treadmill (Life Fitness $^{\circledR}$, model $9700 \mathrm{HR}^{\circledR}$, Fort Mill, Tennessee, USA); finally, 5 minutes of smooth movements, stretching and breathing. A cardiac monitor (Oregon Scientific SE128 ${ }^{\circledR}$, Portland-Oregon), was used individually, according to the HR of each patient every three minutes in all sessions. Each session was conducted under supervision, which promoted safety and accuracy in prescribing individualized training (50-50\% of MaxHR), and if necessary, adjusting for the training zone.

\section{Control group (CG)}

The CG continued their usual activities during the study period, without getting involved in exercise. Patients' follow-up was conducted by telephone every 15 days to assure that the protocol was being followed.

\section{Statistical analysis}

The Kolmogorov-Smirnov test was used to test data normality. Categorical data were expressed as frequency and percentage, whereas continuous data were described as mean and standard deviation. Between-group comparisons (TG x CG) were made at baseline using the unpaired Student's t-test. Withingroup comparisons (pre-training vs post-training) and intergroup comparisons were conducted by the Split-Plot ANOVA, followed by the Bonferroni low or null correlation; post hoc test. The Pearson correlation coefficients (r) used in the study were: $0<\mathrm{r}<0.25$ : low or null correlation; $0.25<\mathrm{r}<0.50$ : weak correlation; $0.50<$ $\mathrm{r}<0.75$ : moderate correlation; and $0.75<\mathrm{r}<1.00$ : strong or perfect correlation. The effect size (ES) was defined as mean Cohen's d greater than 0.2 and lower than 0.5; values between 0.5 and 0.8 were defined as good ES, and values equal to or greater than 0.8 were defined as a large ES. ${ }^{30}$ Sample size was calculated considering an alpha error of $5 \%$, statistical power of $90 \%$, and a sample of 80 subjects. The level of significance was set at 5\% $(\mathrm{p}<0.05)$ and all analyzes were performed using the Statistical Package for the Social Sciences software, version 20.0. (IBM ${ }^{\circledR}$, New York, New York, USA).

\section{Results}

\section{Characteristics of the sample}

Characteristics of the subjects at baseline are described in Table 1. As expected, all volunteers were elderly. Participants in both groups were classified as normal weight or overweight according to the body mass index (BMI) categories. On the other hand, all subjects had a very low to low cardiorespiratory fitness, as compared with the limits established for the elderly population. ${ }^{31}$ The unpaired Student's t-test did not show any significant difference between the groups. All volunteers participated in $100 \%$ of sessions, and no adverse effect was observed during or after the training sessions.

\section{Depressive symptoms and physical pain}

Primary results are shown in Figures 2 and 3. There was a significant reduction in GDS in the TG after 12 weeks of walking training. In contrast both GDS and VAS remained unchanged in the CG (GDS $=3.0 \pm 2.7$ to $3.1 \pm 2.4 ; \mathrm{p}=0.94 ; \mathrm{ES}=-0.03 ; \mathrm{VAS}=4.4 \pm 3.2$ to $4.2 \pm 3.2$; $\mathrm{p}=0.68 ; \mathrm{ES}=0.06$ ).

\section{Quality of life and peak oxygen consumption}

QOL (WHOQoL-OLD and WHOQoL-BREF) (Figure 4) and cardiorespiratory fitness (Table 2) were assessed as secondary outcomes. Regarding QOL, no difference was seen between the groups (TG [WHOQoL-OLD $=66.5$ $\pm 14.0 \%$ to $65.6 \pm 15.9 \%$, $(\mathrm{p}=0.94, \mathrm{ES}=0.06)$; WHOQoL$\mathrm{BREF}=67.8 \pm 11.0$ to $69.4 \pm 10.8(\mathrm{p}=0.21$, ES $=-0.14)]$ and CG [WHOQoL-OLD $=64.7 \pm 12.3$ to $63.2 \pm 13.4$, 
Table 1 - Characteristics and questionnaire scores of participant in the beginning of the study

\begin{tabular}{|c|c|c|c|}
\hline Variables & TG $(n=40)$ & CG $(n=29)$ & $p$ value \\
\hline Age (years) & $68.2(5.2)$ & $65.3(3.8)$ & $0.57^{\mathrm{a}}$ \\
\hline Body mass (kg) & $73.4(12.6)$ & $67.7(14.6)$ & $0.08^{\mathrm{a}}$ \\
\hline Height $(\mathrm{cm})$ & $1.61(0.1)$ & $1.57(0.1)$ & $0.69^{\mathrm{a}}$ \\
\hline BMI $\left(\mathrm{kg} / \mathrm{m}^{2}\right)$ & $28.5(4.9)$ & $27.3(4.5)$ & $0.28^{\mathrm{a}}$ \\
\hline Peak $\mathrm{VO}_{2}\left(\mathrm{ml} \cdot \mathrm{kg}^{-1} \cdot \mathrm{min}^{-1}\right)$ & $23.67(5.2)$ & $23.61(4.9)$ & $0.23^{\mathrm{a}}$ \\
\hline GDS (rating) & $2.7(2.4)$ & $3.0(2.7)$ & $0.89^{\mathrm{a}}$ \\
\hline VAS (cm) & $4.3(3.1)$ & $4.4(3.2)$ & $0.84^{\mathrm{a}}$ \\
\hline WHOQOL-OLD (\%) & $66.5(14.0)$ & $64.7(12.3)$ & $0.84^{\mathrm{a}}$ \\
\hline WHOQOL-BREF (\%) & $67.8(11.0)$ & $67.1(10.6)$ & $0.78^{\mathrm{a}}$ \\
\hline Physical activity ( $\mathrm{min} / \mathrm{sem}$ ) & 215 (91.9) & $280(949.5)$ & $0.45^{\mathrm{a}}$ \\
\hline
\end{tabular}

TG: training group; CG: control group; BMI: body mass index; GDS: geriatric depression scale; VAS: visual analogue scale; SD: standard deviation; a: unpaired Student's t test; WHOQOL: World Health Organization Quality of Life.

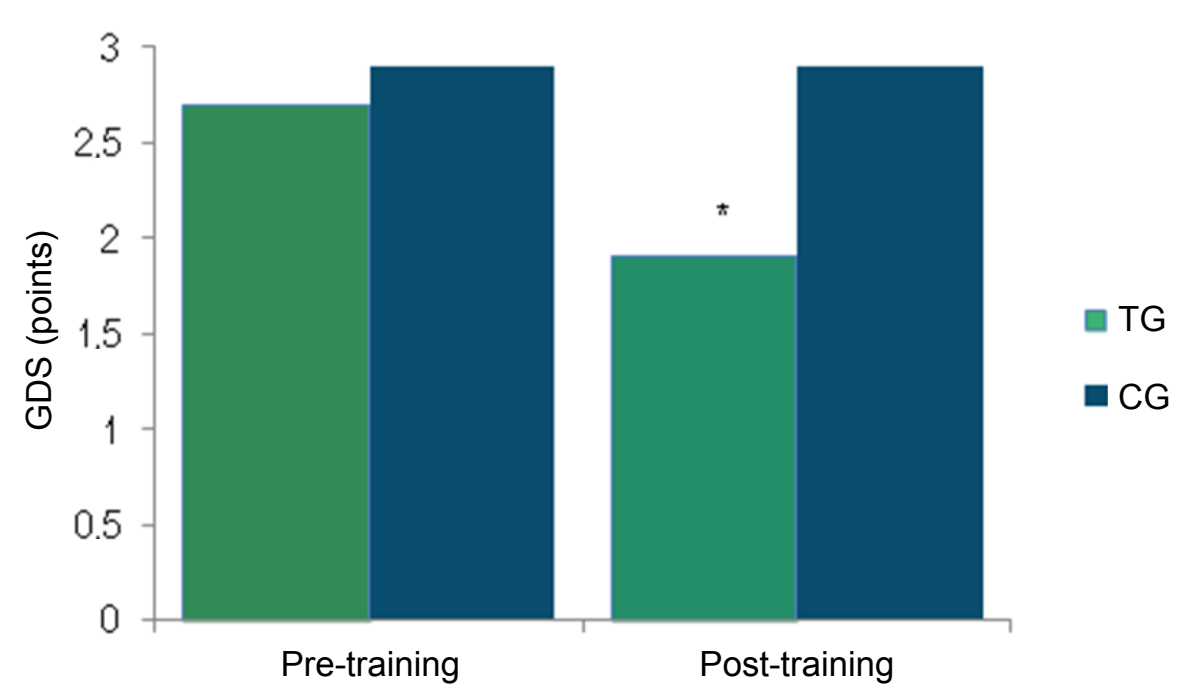

Figure 2 - Geriatric depression scale scores before and after 12 weeks of walking training. GDS: geriatric depression scale; TG: training group; CG: control group; $p=0.04^{*}$ (Student's t-test).

$(\mathrm{p}=0.52, \mathrm{TE}=0.11)] ;$ WHOQoL-BREF $=67.1 \pm 10.6$ to 64.3 $\pm 11.9(\mathrm{p}=0.16, \mathrm{ES}=0.24)$. Similarly, peak $\mathrm{VO}_{2}$ did not show significant differences between the groups before and after the training: TG $(23.67 \pm 5.18$ to $24.46 \pm 5.62)$ and CG (23.61 \pm 4.86 to $23.57 \pm 4.65)$.
Correlation between depressive symptoms and physical pain

Figure 5 illustrates the results of the Pearson analysis. There was a positive, moderate correlation between GDS and VAS $(\mathrm{r}=0.30 ; \mathrm{p}=0.05)$. 


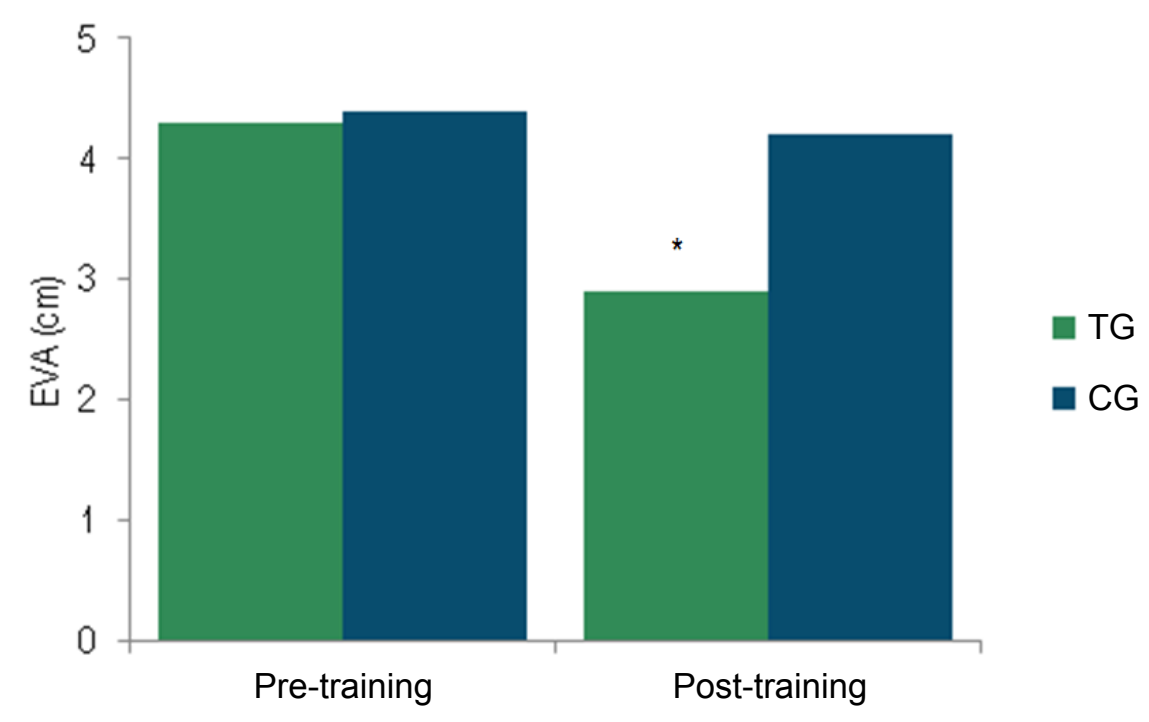

Figure 3 - Visual analogue scale scores before and after 12 weeks of walking training. EVA: visual analogue scale; TG: training group; CG: control group; $p=0.006^{*}$ (Student's $t$-test).

\section{Discussion}

The main findings of the present study indicate that a moderate intensity, 12-week walking training reduces depressive symptoms and physical pain in elderly individuals. Interestingly, improvements seen occurred despite no change in QOL or aerobic fitness. Besides, Pearson correlation showed a significant association between depressive symptoms and pain score in TG.

Data of the present study are in agreement with the literature, since there is considerable evidence showing that different types of training can reduce depressive symptoms in patients with and without depression. ${ }^{32}$ Nevertheless, it is of note that only some studies evaluated the effect of walking training on depressive symptoms of non-depressive patients. In fact, similarly to our study, in the study by Pereira et al. ${ }^{21}$ elderly patients underwent a moderate-intensity (65-80\% of MaxHR) walking training, carried out three days a week for 10 weeks. According to the authors, participants showed a significant reduction in depressive symptoms after exercise, Branco et al. ${ }^{19}$ reported a $37.5 \%$ reduction in depressive symptoms in elderly individuals undergoing a long-term training period (6 months).

In another scenario, different from the data mentioned above, our study included physical pain as an outcome that could be associated with changes in depressive symptoms. In fact, this hypothesis is supported by many authors that indicated a strong association of physical pain with depressive symptoms, mainly in the context of chronic diseases (e.g., cancer). ${ }^{20,33,34}$

Our results showed that GDS and VAS scores were moderately correlated $(\mathrm{r}=0.30)$, suggesting a relationship between effects of aerobic exercise on depressive symptoms and alleviation of symptoms, i.e., a probable analgesic effect of the trainings, leading to pain reduction in elderly individuals. ${ }^{20,33,34}$

It is worth mentioning that there is little evidence on the impact of physical training on depressive symptoms by pain alleviation, and most studies on the topic have been based on cross-sectional data and physical activity level. Sabiston et al., ${ }^{34}$ for example, observed that physical pain was positively associated with depressive symptoms, and that higher levels of exercise had an inhibitory effect on this association in elderly breast cancer patients.

Regarding the exercise protocol used in this study, volunteers of the study by Pennix et al., ${ }^{20}$ showed a reduction in physical pain and depressive symptoms after a moderate-intensity walking training. This corroborates our findings showing a significant hypoalgesia in response to walking training, since we observed a 35\% reduction in overall pain in our volunteers. These results 

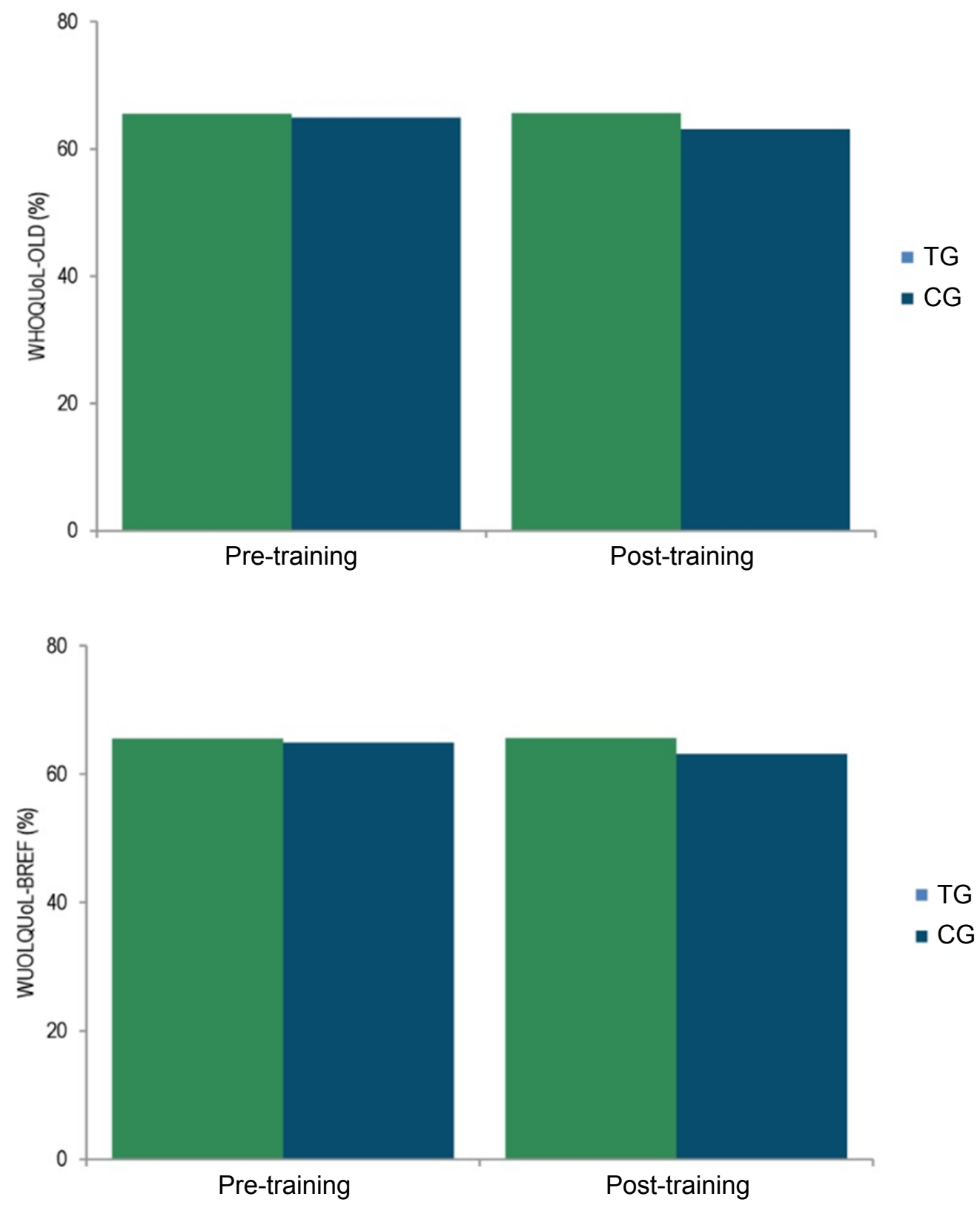

Figure 4 - Results of WHOQoL-OLD and WHOQoL-BREF before and after 12 weeks of walking training. GDS: geriatric depression scale; VAS: visual analogue scale; TG: training group; CG: control group.

are supported by previous studies showing that aerobic exercise seems to increase pain threshold in response to several factors, including ischemic pain, compression and thermal stimulus. ${ }^{35,36}$

In addition, our findings seem to have a broad applicability in clinical practice, since evidence has shown that even healthy elderly subjects undergoing walking training can develop physical pain in an 18-month period. Therefore, our data suggest that a three-month program of moderate-intensity walking training has a protective role on chronic physical pain in healthy elderly individuals..$^{20,22,35}$ Interestingly, body pain and depressive symptoms are two domains of the WHOQoL-OLD and WHOQoL-BREF questionnaires. Nevertheless, although both parameters were reduced in response to our exercise protocol, QOL remained unchanged in the TG. In 
Table 2 - Peak $\mathrm{VO}_{2}$ prior to and after training period (12 weeks)

\begin{tabular}{llcc}
\hline \multicolumn{2}{c}{ Peak VO $\left(\mathrm{ml}_{\mathbf{k g}} \mathbf{k g}^{-1} \mathrm{~min}^{1}\right)$} \\
\hline Groups & Pre-training & Post-training & p value \\
\hline Training & $23.67 \pm 5.18$ & $24.46 \pm 5.62$ & $0.06^{\mathrm{a}}$ \\
Control & $23.61 \pm 4.86$ & $23.57 \pm 4.64$ & $0.95^{\mathrm{a}}$ \\
\hline
\end{tabular}

Peak $\mathrm{VO}_{2}$ : maximum oxygen consumption; a: unpaired Student's $t$ test.

GDS: geriatric depression scale; VAS: visual analogue scale; TG: training group; CG: control group.

contrast, several studies have shown an improvement in QOL of adults undergoing walking training protocols. ${ }^{37-39}$ However, it is worth mentioning that these studies have involved patients with different comorbidities (e.g. hypertension, diabetes), without evaluating other covariates, such as body pain. Also, QOL has not been used as an inclusion criterion.

Although different methods were used in the studies, it is of note that our sample showed higher QOL scores $(\sim 65)$ compared with other studies, ${ }^{37}$ limiting the effects of exercise on these parameters. Besides, evidence has suggested that physical function can be a determining factor in QOL. ${ }^{38,40}$ Since aerobic capacity remained unchanged in the present study, it is possible to infer that this phenomenon is associated with the lack of changes in QOL scores.

\section{Limitations}

This study has some limitations that should be consider to contribute to a better inference from the data. First, the lack of specific physical examinations (muscle and fat mass) that could lead to a better understanding of the relationship between walking training and QOL, as well as the inclusion of other exercise groups, walking training strategies and exercise protocols.

\section{Conclusions}

Our findings indicate that a moderate-intensity walking training with three-month duration can improve depressive symptoms and physical pain in elderly individuals without necessarily changing QOL and aerobic fitness. 


\section{Acknowledgements}

We thank our academic colleagues from the Division of Cardiology of the Federal University of São Paulo and our colleagues from the University of Mogi das CruzesUMC, Physical Education course.

\section{Author contributions}

Conception and design of the research: Alabarse SL, Coelho Júnior HJ, Asano RY, Luna Filho B, Oliveira Filho JA. Acquisition of data: Alabarse SL, Oliveira Filho JA. Analysis and interpretation of the data: Alabarse SL, Coelho Júnior HJ, Asano RY, Oliveira Filho JA. Statistical analysis: Alabarse SL, Coelho Júnior HJ, Luna Filho B, Oliveira Filho JA. Obtaining financing: Alabarse SL, Oliveira Filho JA. Writing of the manuscript: Alabarse SL, Coelho Júnior HJ, Asano RY, Santos WC, Oliveira Filho JA. Critical revision of the manuscript for intellectual content: Alabarse SL, Coelho Júnior HJ, Oliveira Filho JA. Supervision / as the major investigador: Alabarse SL, Oliveira Filho JA.

\section{Potential Conflict of Interest}

No potential conflict of interest relevant to this article was reported.

\section{Sources of Funding}

This study was funded by CAPES.

\section{Study Association}

This article is part of the thesis of Doctoral submitted by Silvio Lopes Alabarse, from Universidade Federal de São Paulo (Unifesp).

\section{Ethics approval and consent to participate}

This study was approved by the Ethics Committee of the Universidade Federal de São Paulo under the protocol number $0562 / 11$. All the procedures in this study were in accordance with the 1975 Helsinki Declaration, updated in 2013. Informed consent was obtained from all participants included in the study.

\section{References}

1. United Nations. World Population Prospects: The 2015 Revision. Key Findings and Advance Tables 2015 [Internet]. New York: United Nations; 2015 [citado 4 abr. 2017]. Disponível em: https://esa.un.org/unpd/ wpp/publications/files/key_findings_wpp_2015.pdf.

2. Coelho Junior HJ, Aguiar Sda S, Goncalves Ide O, Sampaio RA, Uchida MC, Moraes MR, et al. Sarcopenia is Associated with High Pulse Pressure in Older Women. J Aging Res. 2015;2015:109824.

3. Sewo Sampaio PY, Sampaio RA, Coelho Junior HJ, Teixeira LF, Tessutti VD, Uchida MC, et al. Differences in lifestyle, physical performance and quality of life between frail and robust Brazilian community-dwelling elderly women. Geriatr Gerontol Int. 2016;16(7):829-35.

4. Queiroz AC, Kanegusuku H, Forjaz CL. Effects of resistance training on blood pressure in the elderly. Arq Bras Cardiol. 2010;95(1):135-40.

5. Xavier FM, Ferraz MP, Marc N, Escosteguy NU, Moriguchi EH. Elderly people's definition of quality of life. Bras J Psyquiatry. 2003;25(1):31-9.

6. Hao G, Bishwajit G, Tang S, Nie C, Ji L, Huang R. Social participation and perceived depression among elderly population in South Africa. Clin Interv Aging. 2017 Jun 23;12:971-6.

7. Morley JE, Vellas B, van Kan GA, Anker SD, Bauer JM, Bernabei R, et al. Frailty consensus: a call to action. J Am Med Dir Assoc. 2013;14(6):392-7.

8. American College of Sports Medicine. American College of Sports Medicine position stand. Progression models in resistance training for healthy adults. Med Sci Sports Exerc. 2009;41(3):687-708.

9. Garber CE, Blissmer B, Deschenes MR, Franklin BA, Lamonte MJ, Lee IM, et al. American College of Sports Medicine position stand. Quantity and quality of exercise for developing and maintaining cardiorespiratory, musculoskeletal, and neuromotor fitness in

apparently healthy adults: guidance for prescribing exercise. Med Sci Sports Exerc. 2011;43(7):1334-59.

10. Ossowski ZM, Skrobot W, Aschenbrenner P, Cesnaitiene VJ, Smaru M. Effects of short-term Nordic walking training on sarcopenia-related parameters in women with low bone mass: a preliminary study. Clin Interv Aging. 2016 Nov 30;11:1763-71.

11. Asano RY, Sales MM, Browne RA, Moraes JF, Coelho Júnior HJ, Moraes MR, et al. Acute effects of physical exercise in type 2 diabetes: A review. World J Diabetes. 2014;5(5):659-65.

12. Pescatello LS, Franklin BA, Fagard R, Farquhar WB, Kelley GA, Ray CA. American College of Sports Medicine position stand. Exercise and hypertension. Med Sci Sports Exerc. 2004;36(3):533-53.

13. Eckel RH, Jakicic JM, Ard JD, de Jesus JM, Houston Miller N, Hubbard VS, et al. 2013 AHA / ACC guideline on lifestyle management to reduce cardiovascular risk: a report of the American College of Cardiology/ American Heart Association Task Force on Practice Guidelines. J Am Coll Cardiol. 2014;63(25 Pt B):2960-84.

14. American College of Sports Medicine, Chodzko-Zajko WJ, Proctor DN, Fiatarone Singh MA, Minson CT, Nigg CR, et al. American College of Sports Medicine position stand. Exercise and Physical Activity for Older Adults. Med Sci Sports Exerc. 2009;41(7):1510-30.

15. Nelson ME, Rejeski WJ, Blair SN, Duncan PW, Judge JO, King AC, et al. Physical activity and public health in older adults: recommendation from the American College of Sports Medicine and the American Heart Association. Med Sci Sports Exerc. 2007;39(8):1435-45.

16. Culos-Reed SN, Stephenson L, Doyle-Baker PK, Dickinson JA. Mall walking as a physical activity option: results of a pilot project. Can J Aging. 2008;27(1):81-7. 
17. Tully MA, Cupples ME, Chan WS, McGlade K, Young IS. Brisk walking, fitness, and cardiovascular risk: a randomized controlled trial in primary care. Prev Med. 2005;41(2):622-8

18. Murphy M, Nevill A, Neville C, Biddle S, Hardman A. Accumulating brisk walking for fitness, cardiovascular risk, and psychological health. Med Sci Sports Exerc. 2002;34(9):1468-74.

19. Branco JC, Jansen K, Sobrinho JT, Carrapatoso S, Spessato B, Carvalho $\mathrm{J}$, et al. Physical benefits and reduction of depressive symptoms among the elderly: results from the Portuguese "National Walking Program". Cien Saude Colet. 2015;20(3):789-95

20. Penninx BW, Rejeski WJ, Pandya J, Miller ME, Di Bari M, Applegate WB, et al. Exercise and depressive symptoms a comparison of aerobic and resistance exercise effects on emotional and physical function in older persons with high and low depressive symptomatology. J Gerontol B Psychol Sci Soc Sci. 2002;57(2):P124-32.

21. Pereira DS, de Queiroz BZ, Miranda AS, Rocha NP, Felício DC, Mateo EC, et al. Effects of physical exercise on plasma levels of brain-derived neurotrophic factor and depressive symptoms in elderly women--a randomized clinical trial. Arch Phys Med Rehabil. 2013;94(8):1443-50.

22. Tanaka R, Ozawa J, Kito N, Moriyama H. Efficacy of strengthening or aerobic exercise on pain relief in people with knee osteoarthritis: a systematic review and meta-analysis of randomized controlled trials Clin Rehabil. 2013;27(12):1059-71.

23. Hagströmer M, Oja P, Sjöström M. The International Physical Activity Questionnaire (IPAQ): a study of concurrent and construct validity. Public Health Nutr. 2006;9(6):755-62.

24. Marx FC, Oliveira LM, Bellini CG, Ribeiro MCC. Translation and cultural validation of the Lequesne's algofunctional questionnaire for osteoarthritis of knee and hip for portuguese language. Rev Bras Reumatol. 2006;46(4):253-60

25. Fleck MP, Chachamovich E, Trentini C. Development and validation of the Portuguese version of the WHOQOL-OLD module. Rev Saude Publica. 2006;40(5):785-91.

26. Fleck MP, Louzada S, Xavier M, Chachamovich E, Vieira G, Santos L, et al. Application of the Portuguese version of the abbreviated instrument of quality life WHOQOL-bref. Rev Saude Publica. 2000;34(2):178-83.

27. Almeida OP, Almeida SA. Short versions of the geriatric depression scale: a study of their validity for the diagnosis of a major depressive episode according to ICD-10 and DSM-IV. Int J Geriatr Psychiatry. 1999;14(10):858-65.
28. Paradela EM, Lourenco RA, Veras RP. Validation of geriatric depression scale in a general outpatient clinic. Rev Saude Publica. 2005;39(6):918-23.

29. Rapo-Pylkko S, Haanpaa M, Liira H. Subjective easiness of pain assessment measures in older people. Arch Gerontol Geriatr. 2016 JulAug;65:25-8.

30. Kraemer HC, Kupfer DJ. Size of treatment effects and their importance to clinical research and practice. Biol Psychiatry. 2006;59(11):990-6.

31. Herdy AH, Caixeta A. Brazilian Cardiorespiratory Fitness Classification Based on Maximum Oxygen Consumption. Arq Bras Cardiol. 2016;106(5):389-95.

32. Park SH, Han KS, Kang CB. Effects of exercise programs on depressive symptoms, quality of life, and self-esteem in older people: a systematic review of randomized controlled trials. Appl Nurs Res. 2014;27(4):219-26.

33. Kelleher C, Hickey A, Conroy R, Doyle F. Does pain mediate or moderate the relationship between physical activity and depressive symptoms in older people? Findings from The Irish Longitudinal Study on Ageing (TILDA). Health Psychol Behav Med. 2014;2(1):785-97.

34. Sabiston CM, Brunet J, Burke S. Pain, movement, and mind: does physical activity mediate the relationship between pain and mental health among survivors of breast cancer? Clin J Pain. 2012;28(6):489-95.

35. Hoffman MD, Hoffman DR. Does aerobic exercise improve pain perception and mood? A review of the evidence related to healthy and chronic pain subjects. Curr Pain Headache Rep. 2007;11(2):93-7.

36. Koltyn KF. Exercise-induced hypoalgesia and intensity of exercise. Sports Med. 2002:32(8):477-87.

37. Bello AI, Owusu-Boakye E, Adegoke BO, Adjei DN. Effects of aerobic exercise on selected physiological parameters and quality of life in patients with type 2 diabetes mellitus. Int J Gen Med. 2011;4:723-7.

38. Maruf FA, Akinpelu AO, Salako BL. Self-reported quality of life before and after aerobic exercise training in individuals with hypertension: a randomised-controlled trial. Appl Psychol Health Well Being. 2013;5(2):209-24.

39. Reid KJ, Baron KG, Lu B, Naylor E, Wolfe L, Zee PC. Aerobic exercise improves self-reported sleep and quality of life in older adults with insomnia. Sleep Med. 2010;11(9):934-40.

40. Canuto Wanderley FA, Oliveira NL, Marques E, Moreira P, Oliveira J, Carvalho J. Aerobic versus resistance training effects on health-related quality of life, body composition, and function of older adults. J Appl Gerontol. 2015;34(3):NP143-65. 